\title{
Low-energy constants from ALEPH hadronic tau decay data
}

\author{
Diogo Boito \\ Instituto de Física de São Carlos, Universidade de São Paulo, CP 369, 13560-970, São Carlos, \\ SP, Brazil \\ E-mail: boitodifsc.usp.br
}

\section{Anthony Francis}

Dept. of Physics and Astronomy, York Univ., Toronto, ON Canada M3J 1P3

E-mail: afrancis.heplategooglemail.com

\section{Maarten Golterman*}

Dept. of Physics and Astronomy, San Francisco State Univ., San Francisco, CA 94132, USA

E-mail: maartenesfisu.edu

\section{Renwick Hudspith}

Dept. of Physics and Astronomy, York Univ., Toronto, ON Canada M3J 1P3

E-mail: renwick.james.hudspith@gmail.com

\section{Randy Lewis}

Dept. of Physics and Astronomy, York Univ., Toronto, ON Canada M3J 1P3

E-mail: randy. lewis @yorku.ca

\section{Kim Maltman}

Dept. of Mathematics and Statistics, York Univ., Toronto, ON Canada M3J 1P3

CSSM, Univ. of Adelaide, Adelaide, SA 5005 Australia

E-mail: kmaltman@yorku.ca

\section{Santiago Peris}

Dept. of Physics, Univ. Autònoma de Barcelona, E-08193 Bellaterra, Barcelona, Spain

E-mail: periseifae.es

We determined the NLO chiral low-energy constant $L_{10}$, and various combinations of NNLO chiral low-energy constants employing recently revised ALEPH results for the non-strange vector $(V)$ and axial-vector $(A)$ hadronic tau decay distributions and recently updated RBC/UKQCD lattice data for the non-strange $V-A$ two-point function. In this talk, we explain the ingredients of this determination. Our errors are at or below the level expected for contributions of yet higher order in the chiral expansion, suggesting that our results exhaust the possibilities of what can be meaningfully achieved in an NNLO analysis.

The 8th International Workshop on Chiral Dynamics, CD2015 ***

29 June 2015 - 03 July 2015

Pisa,Italy

\footnotetext{
* Speaker.
} 


\section{Introduction}

In a recent paper [1] we revisited the determination of the next-to-leading order (NLO) lowenergy constant (LEC) $L_{10}$, and various combinations of NNLO LECs (denoted by $C_{i}$ ) from hadronic $\tau$ decays, which we had previously [2,3] extracted from OPAL data [4]. The motivation for doing so is that the ALEPH data for the hadronic spectral functions measured from $\tau$ decays has recently been revised [5], to correct for the inadvertent omission of unfolding correlations from the covariance matrix [6]. The ALEPH data are more precise, and thus lead to values for these LECs with smaller errors.

These data were analyzed previously in Ref. [7], the main goal being an extraction of $\alpha_{s}\left(m_{\tau}^{2}\right)$, and we will make use of the results of that analysis here. These data are used in finite-energy sum rules (FESRs) involving the non-strange $V-A$ combinations of vector two-point correlators and spectral functions. We also employ flavor-breaking inverse moment FESRs in the $V$ and $V \pm$ $A$ channels, following the pioneering work of Ref. [8], since these provide access to additional combinations of NNLO LECs. For these we also need data from strange hadronic tau decays. Results for the main exclusive modes were taken from BaBar and Belle, with ALEPH data used for the remainder (details, including references, can be found in Ref. [3]).

Finally, we also employ lattice data $[1,9,10,11,12]$, since the lattice allows us to vary the pseudo Nambu-Goldstone boson masses, and thus to separate, in particular, $L_{10}$ from $C_{12}-C_{61}+$ $C_{80}$ and $C_{13}-C_{62}+C_{81}$. The values of $L_{5}$ and $L_{9}$, which also contribute to the $V$ and $A$ two-point correlators [13], are input to our analysis, and taken from Refs. [14] and [15], respectively.

The summary of our work which we will present here will be brief. All details can be found in Ref. [1] and references therein.

\section{Sum rules}

We begin with the non-strange $V-A$ vacuum polarization sum rules. Define

$$
\bar{\Pi}_{V-A}^{(w)}\left(Q^{2}\right)=\int_{0}^{\infty} d s w\left(s / s_{0}\right) \frac{\rho_{V}(s)-\bar{\rho}_{A}(s)}{s+Q^{2}}, \quad 0<s_{0} \leq m_{\tau}^{2},
$$

with $\rho_{V / A}$ the $V / A$ non-strange spectral function, with a bar indicating that the contribution from the pion has been omitted, and where $w(x)$ is a polynomial. Then [2]

$$
\begin{aligned}
-8 L_{10}^{\mathrm{eff}} & \equiv \bar{\Pi}_{V-A}(0) \\
& =\bar{\Pi}_{V-A}^{\left(w_{2}\right)}(0)+\frac{4 f_{\pi}^{2}}{s_{0}}(1-\underbrace{\frac{17 \alpha_{s}^{2}\left(s_{0}\right) m_{u, d}^{2}\left(s_{0}\right)}{16 \pi^{4} f_{\pi}^{2}}-\frac{m_{\pi}^{2}}{2 s_{0}}\left(1+O\left(\alpha_{s}\right)\right)}_{\text {numerically negligible }}) \\
-16 C_{87}^{\mathrm{eff}} & =\bar{\Pi}_{V-A}^{\prime}(0),
\end{aligned}
$$

with the choice of weight

$$
w_{2}(x)=(1-x)^{2}
$$

for the polynomial $w(x)$ in Eq. (2.1), defines effective LECs $L_{10}^{\text {eff }}$ and $C_{87}^{\text {eff }}$, related to, but not equal to, $L_{10}$ and $C_{87}$ (see below). The expression with the weight $w_{2}$ leads to smaller error on $L_{10}^{\text {eff }}$, because 
the second term is known very precisely (even if we neglect the terms labeled as "numerically negligible"). We will therefore employ this expression to obtain the numerical value of $L_{10}^{\text {eff }}$.

We extract $\bar{\Pi}_{V-A}^{(w)}\left(Q^{2}\right)$ from the data using the split

$$
\begin{aligned}
\bar{\Pi}_{V-A}^{(w)}\left(Q^{2}\right)= & \sum_{\text {bins }<s_{\mathrm{sw}}} w_{\mathrm{av}}\left(\frac{s_{\mathrm{bin}}}{s_{\mathrm{sw}}}\right) \frac{\rho_{V}\left(s_{\mathrm{bin}}\right)-\bar{\rho}_{A}\left(s_{\mathrm{bin}}\right)}{s_{\mathrm{bin}}+Q^{2}} \\
& +\int_{s_{\mathrm{sw}}}^{\infty} d s w\left(\frac{s}{s_{\mathrm{sw}}}\right) \frac{\rho_{V}^{\mathrm{DV}}(s)-\rho_{A}^{\mathrm{DV}}(s)}{s+Q^{2}}
\end{aligned}
$$

where the first term is determined directly from the data, ${ }^{1}$ and the second term is evaluated using the parametrization

$$
\rho_{T}(s)=e^{-\delta_{T}-\gamma_{T} s} \sin \left(\alpha_{T}+\beta_{T} s\right), \quad T \in\{V, A\}
$$

Values for the parameters in Eq. (2.5) were obtained from fits to weighted moments of the ALEPH spectral functions in Ref. [7]. Furthermore, we took $s_{\mathrm{sw}}=1.55 \mathrm{GeV}^{2}$, and all correlations, including those between the first and second terms in Eq. (2.4) were taken into account. ${ }^{2}$ While the contribution of the second term in Eq. (2.4) is small, including it allows us to check on the contribution from duality violations to $L_{10}^{\text {eff }}$ and $C_{87}^{\text {eff }}$ quantitatively.

For the flavor-breaking inverse-moment sum rules, we define

$$
\Delta \Pi_{T}\left(Q^{2}\right) \equiv \Pi_{u d ; T}\left(Q^{2}\right)-\Pi_{u s ; T}\left(Q^{2}\right), \quad T \in\{V, A, V \pm A\},
$$

and the sum rules of interest then take the form $[1,8]$

$$
\begin{aligned}
\Delta \Pi_{V}(0) & =\int_{4 m_{\pi}^{2}}^{s_{0}} d s \frac{w\left(s / s_{0}\right)}{s} \Delta \rho_{V}(s)+\mathrm{OPE} \\
\Delta \bar{\Pi}_{V \pm A}(0) & =\int_{4 m_{\pi}^{2}}^{s_{0}} d s \frac{w\left(s / s_{0}\right)}{s} \Delta \bar{\rho}_{V \pm A}(s) \pm\left(\frac{2 f_{K}^{2}}{m_{K}^{2}}\left(1-w\left(\frac{m_{K}^{2}}{s_{0}}\right)\right)-(K \rightarrow \pi)\right)+\mathrm{OPE} \\
w(x) & = \begin{cases}(1-x)^{3} \\
(1-x)^{3}\left(1+x+\frac{1}{2} x^{2}\right) & \text { (DK weight) }\end{cases}
\end{aligned}
$$

In this case, we neglected the contribution from duality violations, because of the fact that both weights are triply pinched at $s=s_{0}$ and the additional $1 / s$ suppression of the region near $s=s_{0}$. We included perturbative and non-perturbative contributions from the operator product expansion (OPE) with conservative estimates of the systematic errors for these contributions. As clearly the left-hand side of these equations is independent of $s_{0}$, this allowed us to carry out a selfconsistency check by considering the $s_{0}$ dependence of the right-hand side, which we did on the interval $2 \mathrm{GeV}^{2}<s_{0}<m_{\tau}^{2}$. We found that $s_{0}$-independence is well satisfied within errors. In addition, we checked that our results are independent of which of the two weights in Eq. (2.7) was employed.

\footnotetext{
${ }^{1}$ The subscript "av" on $w$ indicated that we average the weight $w$ over the width of each bin.

${ }^{2}$ At $Q^{2}=0$ there is no discernible sensitivity to the precise value of $s_{\mathrm{sw}}$.
} 
We summarize the results we find from employing these sum rules:

$$
\begin{aligned}
L_{10}^{\text {eff }} & =-6.446(50) \times 10^{-3}, \\
C_{87}^{\mathrm{eff}} & =8.38(18) \times 10^{-3} \mathrm{GeV}^{-2}, \\
\Delta \Pi_{V}(0) & =0.0224(9) \\
\Delta \bar{\Pi}_{A}(0) & =0.113(8) \\
\Delta \bar{\Pi}_{V+A}(0) & =0.0338(10) \\
\Delta \bar{\Pi}_{V-A}(0) & =0.0111(11)
\end{aligned}
$$

\section{Connection with chiral perturbation theory}

The connection between the effective LECs of Eq. (2.2) and chiral perturbation theory (ChPT) is given by [13]

$$
\begin{aligned}
L_{10}^{\mathrm{eff}}= & L_{10}^{r}\left(1-4\left(2 \mu_{\pi}+\mu_{K}\right)\right)-2\left(2 \mu_{\pi}+\mu_{K}\right) L_{9}^{r}-\frac{1}{8} \hat{R}_{\pi K}(\mu, 0) \\
& -4 m_{\pi}^{2}\left(C_{12}^{r}-C_{61}^{r}+C_{80}^{r}\right)-4\left(2 m_{K}^{2}+m_{\pi}^{2}\right)\left(C_{13}^{r}-C_{62}^{r}+C_{81}^{r}\right), \\
C_{87}^{\mathrm{eff}}= & C_{87}^{r}-\frac{1}{64 \pi^{2} f_{\pi}^{2}}\left(1-\log \frac{\mu^{2}}{m_{\pi}^{2}}+\frac{1}{3} \log \frac{m_{K}^{2}}{m_{\pi}^{2}}\right) L_{9}^{r}-\frac{1}{16} \hat{R}_{\pi K}^{\prime}(\mu, 0),
\end{aligned}
$$

where $\mu_{P}=\left(m_{P}^{2} /\left(32 \pi^{2} f_{\pi}^{2}\right)\right) \log \left(m_{P}^{2} / \mu^{2}\right)$, and the functions $\hat{R}_{\pi K}$ and $\hat{R}_{\pi K}^{\prime}$ are known functions of the renormalization scale $\mu$ [1], $m_{\pi}$, and $m_{K}$. Below, we will take $\mu=770 \mathrm{MeV}$, and $L_{9}^{r}=5.93(43) \times$ $10^{-3}[15]$.

Clearly, the only way to disentangle $L_{10}^{r}$ from the combinations $C_{12}^{r}-C_{61}^{r}+C_{80}^{r}$ and $C_{13}^{r}-$ $C_{62}^{r}+C_{81}^{r}$ is to vary the pion (or the kaon) mass, and this can only be done using Lattice QCD. To this end, we employ three RBC/UKQCD ensembles, two with $1 / a=1.379(7) \mathrm{GeV}$ and $m_{\pi}=172$ or $250 \mathrm{MeV}$, and one with $1 / a=1.785(5) \mathrm{GeV}$ and $m_{\pi}=340 \mathrm{MeV}[1,9,10,11,12]$.

For the $\Delta \Pi_{V}(0)$ and $\Delta \bar{\Pi}_{V \pm A}(0)$, the ChPT expressions, substituting physical masses and decay constants, setting $\mu=770 \mathrm{MeV}$, and defining $\Delta_{\pi K} \equiv 32\left(m_{K}^{2}-m_{\pi}^{2}\right)=7.238 \mathrm{GeV}^{2}$, are given by

$$
\begin{aligned}
\Delta \Pi_{V}(0) & =0.00775-0.7218 L_{5}^{r}+1.423 L_{9}^{r}+1.062 L_{10}^{r}+\Delta_{\pi K} C_{61}^{r}, \\
\Delta \bar{\Pi}_{V+A}(0) & =0.00880-0.7218 L_{5}^{r}+1.423 L_{9}^{r}+\Delta_{\pi K}\left(C_{12}^{r}+C_{61}^{r}+C_{80}^{r}\right) \\
\Delta \bar{\Pi}_{V-A}(0) & =0.00670-0.7218 L_{5}^{r}+1.423 L_{9}^{r}+2.125 L_{10}^{r}-\Delta_{\pi K}\left(C_{12}^{r}-C_{61}^{r}+C_{80}^{r}\right) .
\end{aligned}
$$

Using the value $L_{5}^{r}=0.84(38) \times 10^{-3}$ from Ref. [14] and the value for $L_{9}^{r}$ quoted above, these expressions allow us to obtain $C_{12}^{r}+C_{61}^{r}+C_{80}^{r}$ from $\Delta \bar{\Pi}_{V+A}(0)$, then from $\Delta \bar{\Pi}_{V-A}(0), L_{10}^{\text {eff }}$ and the lattice we get $C_{12}^{r}-C_{61}^{r}+C_{80}^{r}$ and $C_{13}^{r}-C_{62}^{r}+C_{81}^{r}$, while $\Delta \Pi_{V}(0)$ gives us direct access to $C_{61}^{r}$.

Taking all correlations into account, this set up yields the following results for $L_{10}^{r}$ and several 
(combinations of) NNLO LECs ${ }^{3}$

$$
\begin{aligned}
L_{10}^{r}= & -3.50(17) \times 10^{-3}, \\
C_{12}^{r}+C_{61}^{r}+C_{80}^{r}= & 2.37(16) \times 10^{-3} \mathrm{GeV}^{-2}, \\
C_{12}^{r}-C_{61}^{r}+C_{80}^{r}= & -0.56(15) \times 10^{-3} \mathrm{GeV}^{-2}, \\
C_{13}^{r}-C_{62}^{r}+C_{81}^{r}= & 0.46(9) \times 10^{-3} \mathrm{GeV}^{-2}, \\
C_{61}^{r}= & 1.46(15) \times 10^{-3} \mathrm{GeV}^{-2}, \\
C_{12}^{r}+C_{80}^{r}= & 0.90(9) \times 10^{-3} \mathrm{GeV}^{-2}, \\
C_{87}^{r}= & 5.10(22) \times 10^{-3} \mathrm{GeV}^{-2},
\end{aligned}
$$

where $C_{87}^{r}$ was obtained directly from Eq. (3.2).

\section{Conclusion}

The results presented in Eq. (3.4) constitute our best results for these (combinations of) LECs. They are consistent with the results obtained earlier from OPAL data in Refs. [2, 3, 9], but the errors are smaller. Since our analysis method was the same, this is due to the higher precision of the revised ALEPH data.

Of course, one wonders whether it is possible to do better. However, there is a systematic effect due to the neglect of $\mathrm{N}^{3} \mathrm{LO}$ terms in ChPT, which is not included in the errors shown in Eq. (3.4). We estimate that the error due to neglecting higher orders in ChPT is about $6 \%$ for $L_{10}^{r}$, and about $25 \%$ for NNLO LECs. ${ }^{4}$ This means that the precision attainable with an NNLO analysis has been reached.

We note that the combination $C_{13}^{r}-C_{62}^{r}+C_{81}^{r}$ is not smaller in size than the combination $C_{12}^{r}-C_{61}^{r}+C_{80}^{r}$, even though the LECs $C_{13,62,81}^{r}$ are suppressed relative to $C_{12,61,80}^{r}$ in the limit $N_{c} \rightarrow \infty$. Since cancellations in the combination $C_{12}^{r}-C_{61}^{r}+C_{80}^{r}$ take place (note that the values for $C_{12}^{r}+C_{80}^{r}$ and $C_{61}^{r}$ are indeed significantly larger than that for $C_{12}^{r}-C_{61}^{r}+C_{80}^{r}$ ), this does not invalidate the expansion in $1 / N_{c}$; however, it does invalidate the assumption made in Ref. [16] that the combination $C_{13}^{r}-C_{62}^{r}+C_{81}^{r}$ can be set approximately equal to zero. We also note that our parametrization of the duality-violating part of the spectral functions, given in Eq. (2.5), is more general than that employed in Ref. [16] (whereas in Ref. [17] they were neglected altogether), thus avoiding unnecessary additional assumptions. For a detailed discussion of the assumptions underlying the use of Eq. (2.5), we refer to Ref. [18].

Finally, we remark that in Ref. [1] we also determined the coefficients of $Q^{-6}$ and $Q^{-8}$ in the OPE of the $V-A$ two-point function. We find values that differ by about 2.4 standard deviations from those we obtained from the OPAL data in Ref. [2].

\section{Acknowledgments}

DB thanks the Department of Physics of the Universitat Autònoma de Barcelona, and KM and SP thank the Department of Physics and Astronomy at San Francisco State University for

\footnotetext{
${ }^{3}$ For the many details, we refer to Refs. [1, 2, 3].

${ }^{4}$ For a detailed discussion of this, see Ref. [2].
} 
hospitality. MG is supported in part by the US Department of Energy, AF, RH, RL and KM are supported by grants from the Natural Sciences and Engineering Research Council of Canada, and SP is supported by CICYT-FEDER-FPA2014-55613-P, 2014 SGR 1450, the Spanish ConsoliderIngenio 2010 Program CPAN (CSD2007-00042). Propagator inversions for the improved lattice data were performed on the STFC funded "DiRAC" BG/Q system in the Advanced Computing Facility at the University of Edinburgh.

\section{References}

[1] D. Boito, A. Francis, M. Golterman, R. Hudspith, R. Lewis, K. Maltman and S. Peris, arXiv:1503.03450 [hep-ph].

[2] D. Boito, M. Golterman, M. Jamin, K. Maltman and S. Peris, Phys. Rev. D 87094008 (2013) [arXiv:1212.4471 (hep-ph)].172

[3] M. Golterman, K. Maltman and S. Peris, Phys. Rev. D 89, 054036 (2014) [arXiv:1402.1043 [hep-ph]].

[4] K. Ackerstaff et al. [OPAL Collaboration], Eur. Phys. J. C 7, 571 (1999) [arXiv:hep-ex/9808019].

[5] M. Davier, A. Hoecker, B. Malaescu, C. Z. Yuan and Z. Zhang, Eur. Phys. J. C 74, 2803 (2014) [arXiv:1312.1501 [hep-ex]].

[6] D. Boito, O. Catà, M. Golterman, M. Jamin, K. Maltman, J. Osborne and S. Peris, Nucl. Phys. Proc. Suppl. 218, 104 (2011) [arXiv:1011.4426 [hep-ph]].

[7] D. Boito, M. Golterman, K. Maltman, J. Osborne and S. Peris, Phys. Rev. D 91, 034003 (2015) [arXiv:1410.3528 [hep-ph]].

[8] S. Dürr and J. Kambor, Phys. Rev. D 61, 114025 (2000) [hep-ph/9907539].

[9] P. A. Boyle et al., Phys. Rev. D 89, 094510 (2014) [arXiv:1403.6729 [hep-ph]].

[10] Y. Aoki et al. [RBC and UKQCD Collaborations], Phys. Rev. D 83, 074508 (2011) [arXiv:1011.0892 [hep-lat]].

[11] R. Arthur et al. [RBC and UKQCD Collaborations], Phys. Rev. D 87, 094514 (2013) [arXiv:1208.4412 [hep-lat]].

[12] T. Blum et al. [RBC and UKQCD Collaborations], arXiv:1411.7017 [hep-lat].

[13] G. Amorós, J. Bijnens and P. Talavera, Nucl. Phys. B 568, 319 (2000) [hep-ph/9907264].

[14] A. Bazavov et al. [MILC Collaboration], PoS CD 09, 007 (2009) [arXiv:0910.2966 [hep-ph]].

[15] J. Bijnens and P. Talavera, JHEP 0203046 (2002) [hep-ph/0203049].

[16] M. González-Alonso, A. Pich and J. Prades, Phys. Rev. D 78, 116012 (2008) [arXiv:0810.0760 [hep-ph]]; Phys. Rev. D 82, 014019 (2010) [arXiv:1004.4987 [hep-ph]].

[17] C. A. Dominguez, L. A. Hernandez, K. Schilcher and H. Spiesberger, arXiv:1410.3779 [hep-ph].

[18] D. Boito, O. Catà, M. Golterman, M. Jamin, K. Maltman, J. Osborne and S. Peris, Phys. Rev. D 84, 113006 (2011) [arXiv:1110.1127 [hep-ph]]. 